\title{
Controlling resonance energy transfer in nanostructure emitters by positioning near a mirror
}

Dilusha Weeraddana, Malin Premaratne, Sarath D. Gunapala, and David L. Andrews

Citation: The Journal of Chemical Physics 147, 074117 (2017); doi: 10.1063/1.4998459

View online: http://dx.doi.org/10.1063/1.4998459

View Table of Contents: http://aip.scitation.org/toc/jcp/147/7

Published by the American Institute of Physics

\section{Articles you may be interested in}

Temperature dependence of the hydrated electron's excited-state relaxation. II. Elucidating the relaxation mechanism through ultrafast transient absorption and stimulated emission spectroscopy

The Journal of Chemical Physics 147, 074504 (2017); 10.1063/1.4985906

Temporal dynamics of excitonic states with nonlinear electron-vibrational coupling

The Journal of Chemical Physics 147, 074114 (2017); 10.1063/1.4985910

Tracking an electronic wave packet in the vicinity of a conical intersection

The Journal of Chemical Physics 147, 074101 (2017); 10.1063/1.4989462

Temperature dependence of the hydrated electron's excited-state relaxation. I. Simulation predictions of resonance Raman and pump-probe transient absorption spectra of cavity and non-cavity models

The Journal of Chemical Physics 147, 074503 (2017); 10.1063/1.4985905

Transition state theory for activated systems with driven anharmonic barriers

The Journal of Chemical Physics 147, 074104 (2017); 10.1063/1.4997571

A coupled cluster theory with iterative inclusion of triple excitations and associated equation of motion formulation for excitation energy and ionization potential

The Journal of Chemical Physics 147, 074103 (2017); 10.1063/1.4985916

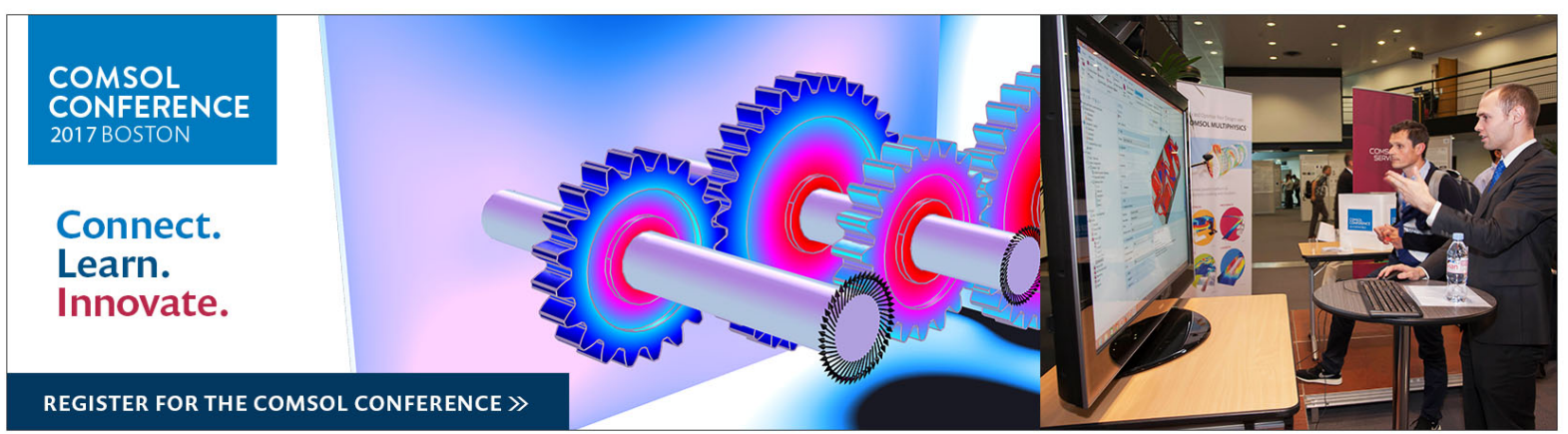




\title{
Controlling resonance energy transfer in nanostructure emitters by positioning near a mirror
}

\author{
Dilusha Weeraddana, ${ }^{1, a)}$ Malin Premaratne, ${ }^{1, b)}$ Sarath D. Gunapala, ${ }^{2, c)}$ \\ and David L. Andrews ${ }^{3, d}$ ) \\ ${ }^{1}$ Advanced Computing and Simulation Laboratory $(A \chi L)$, Department of Electrical and Computer Systems \\ Engineering, Monash University, Clayton, Victoria 3800, Australia \\ ${ }^{2}$ Jet Propulsion Laboratory, California Institute of Technology, Pasadena, California 91109, USA \\ ${ }^{3}$ School of Chemistry, University of East Anglia, Norwich Research Park, Norwich NR4 7TJ, United Kingdom
}

(Received 29 May 2017; accepted 30 July 2017; published online 21 August 2017)

\begin{abstract}
The ability to control light-matter interactions in quantum objects opens up many avenues for new applications. We look at this issue within a fully quantized framework using a fundamental theory to describe mirror-assisted resonance energy transfer (RET) in nanostructures. The process of RET communicates electronic excitation between suitably disposed donor and acceptor particles in close proximity, activated by the initial excitation of the donor. Here, we demonstrate that the energy transfer rate can be significantly controlled by careful positioning of the RET emitters near a mirror. The results deliver equations that elicit new insights into the associated modification of virtual photon behavior, based on the quantum nature of light. In particular, our results indicate that energy transfer efficiency in nanostructures can be explicitly expedited or suppressed by a suitably positioned neighboring mirror, depending on the relative spacing and the dimensionality of the nanostructure. Interestingly, the resonance energy transfer between emitters is observed to "switch off" abruptly under suitable conditions of the RET system. This allows one to quantitatively control RET systems in a new way. Published by AIP Publishing. [http://dx.doi.org/10.1063/1.4998459]
\end{abstract}

\section{INTRODUCTION}

The process of energy transfer plays an extremely important role in the optics and photophysics of solid phase physical systems - specifically, those whose nanoscale structures comprise electronically distinct components with well characterised absorption and emission characteristics. This process is primarily responsible for redirecting or channeling electronic excitation following resonance absorption. Systems exhibiting the effect range from those with an intrinsically complex constitution to others created by nanoscale engineering. In the former connection, it is the significance and effectiveness of the transfer process in biological systems that have led to a surge of interest in the development of nanophotonic applications. In photosynthesis, in particular, the excitation energy is absorbed by pigments in the photosynthetic antennae and subsequently transferred to a reaction center by a series of hops between other chromophore units. ${ }^{1,2}$ In naturally occurring living organisms which perform photosynthesis, it has been found that the light-harvesting efficiency is indeed above $99 \% .^{1,3}$ Although this phenomenon has been studied extensively over many decades, there still exist open questions regarding the underlying mechanism leading to this remarkably high efficiency.

It has already been identified that Resonance Energy Transfer (RET), often also known as the Förster resonance

\footnotetext{
a) Electronic mail: dilusha.weeraddana@monash.edu

b) Electronic mail: malin.premaratne@ monash.edu.

c) Electronic mail: Sarath.D.Gunapala@jpl.nasa.gov

d) Electronic mail: d.1.andrews@uea.ac.uk
}

energy transfer, ${ }^{4}$ is the main energy transfer mechanism in the process of energy transportation in photosynthesis. ${ }^{5}$ Therefore, conducting an in-depth research in RET and being able to control the RET rate would be beneficial to many of the promising applications in nanophotonics, ranging from photovoltaics $^{6-8}$ to bio-medical sensing ${ }^{9,10}$ where molecular distances and interactions are examined. ${ }^{11,12}$

The process of RET occurs in the near-field regime, and it is fully quantum mechanical in nature. ${ }^{13-15}$ The inter-particle interactions are evaluated using Quantum Electrodynamical (QED) theory due to the fact that the minimal- and multipolarcoupling Hamiltonians are many-body Hamiltonians. ${ }^{16,17}$ This is the one advantage of employing a fully quantized approach to study RET systems. In the framework of QED, coupling between bodies is mediated by the exchange of virtual photons, which are not detected. Their lifetime is limited by the time-energy uncertainty principle manifesting in one or more emission-absorption processes. ${ }^{18-20}$

Generally, RET is the dominant energy transfer mechanism between emitters (a donor and an acceptor) in nanometer proximity, where, typical of the Förster theory, the rate has a characteristic $R^{-6}$ distance dependence ( $R$ here is the separation between donor and acceptor molecules). ${ }^{4,21}$ Other means to control a RET system are the spectral properties of the donor and acceptor (donor's emission spectrum and acceptor's absorption spectrum) or by the relative orientations of the transition dipole moments. ${ }^{19,22,23}$ In fact, it is also possible to control the RET rate purely by means of the surrounding environment while leaving the RET pair geometrically and chemically unchanged, by a passive third-body ${ }^{24,25}$ or 
by an auxiliary laser beam impinging on the donor-acceptor system. ${ }^{26-28}$ Further, in cavity QED, inter-particle interactions can be modified due to both the propagating and evanescent modes inside the cavity. ${ }^{29-34}$

However, there are ongoing debates stimulated by modern nanofabrication techniques, about controlling RET purely by means of the nanophotonic environment. Indeed, theory and experiments have revealed both enhanced and inhibited RET rates for many different nanophotonic systems, ranging from plasmonic systems to spasers. ${ }^{35-37}$ Recently, Wubs and Vos studied the Förster resonance energy transfer in nanophotonic media with weak dispersion, ${ }^{38}$ by considering results for the analytical model of a pair of dipole emitters in the vicinity of an ideal mirror. Even though basic principles of RET and control of the RET rate in basic building blocks such as atoms and molecules have been extensively studied, there still exist open questions regarding the mechanisms of controlling the RET phenomenon in nanostructures such as quantum dots (QDs) and nanowires (NWs). As the quantum confinement in nanostructure materials makes them capable of providing unique and superior optical properties, ${ }^{39-41}$ in this study, we specifically focus on RET in nanostructures of different dimensions.

Thus, the main purpose of the present article is to provide new theoretical insights into controlling the rate of RET in nanostructure systems, purely by means of the environment while leaving the RET pair geometrically and chemically untouched. To this end, we have developed a simple model based on the QED framework, by involving the placement of an ideal mirror near the RET nanostructure emitters. We seek to discover what particular effects arise in the matrix element and the transfer rate when input excitation is located in the vicinity of a single mirror, in terms of atomic transition frequency and relative distances, constructing a detailed picture of how individual virtual photon behaviour is modified by the vicinal non-absorbing mirror.

\section{QED FRAMEWORK FOR MOLECULAR INTERACTIONS IN THE VICINITY OF A MIRROR}

\section{A. Theory}

We begin by introducing a suitable quantum framework to describe the quantum level light-matter interaction for RET. Within the QED framework, the light-matter interaction is studied using perturbation theory, to accurately cast the coupling matrix element. ${ }^{13,42}$ The experimentally measurable quantity, $\Gamma_{\text {trans }}$, the energy transfer rate of any photophysical process proceeding from any initial system state $I$ to a final state $F$, can then be determined through Fermi's golden rule, $^{43}$

$$
\Gamma_{\text {tran }}=\frac{2 \pi}{\hbar}\left|M_{F I}^{d}\right|^{2} \rho
$$

in which $\rho$ represents the density of the final system states. The coupling strength between $I$ and $F$ is characterized by the quantum amplitude (coupling matrix element, resonant dipoledipole interaction), $M_{F I}$, formally cast as an infinite series, which is expressed as follows:

$$
M_{F I}=\sum_{q=1}^{\infty}\left\langle F\left|H_{i n t}(\xi)\left(\frac{H_{i n t}(\xi)}{E_{I}-H_{0}}\right)^{q-1}\right| I\right\rangle
$$

where $E_{I}$ is the initial system energy and $H_{0}$ represents the unperturbed system Hamiltonian. The parameter $q$ denotes the power of the radiation-matter interaction Hamiltonian, $H_{\text {int }}(\xi)$, which comprises contributions for each species $\xi$ located at $\mathbf{R}_{\xi}$,

$$
H_{\text {int }}(\xi)=\sum_{\xi}-\mu(\xi) \cdot \mathbf{E}\left(\mathbf{R}_{\xi}\right)
$$

In the above expression, $\boldsymbol{\mu}(\xi)$ is the electric-dipole moment operator and the electric field operator is given by $\mathbf{E}\left(\mathbf{R}_{\xi}\right)$. Further, in the framework of molecular QED, the Hamiltonian for the radiation and matter system can generally be written as $^{13,18}$

$$
H_{\text {total }}=\sum_{\xi} H_{\text {int }}(\xi)+\sum_{\xi} H_{m o l}(\xi)+H_{\text {rad }} .
$$

The operator $H_{m o l}(\xi)$ is the molecular Hamiltonian in the nonrelativistic Born-Oppenheimer approximation, and $H_{\text {rad }}$ is the second-quantized radiation field Hamiltonian. ${ }^{44}$

The eigenstates, $|\tau\rangle$, of a basis Hamiltonian given in Eq. (4) form a composite set that can be expressed by

$$
|\tau\rangle=\left|\operatorname{mat}_{\tau}\right\rangle\left|\operatorname{rad}_{\tau}\right\rangle \equiv\left|\operatorname{mat}_{\tau} ; \operatorname{rad}_{\tau}\right\rangle
$$

$\left|m a t_{\tau}\right\rangle$ in Eq. (5) defines the status of all particles (or molecules, according to the system), comprising a product of state vectors for each matter, and $\left|r a d_{\tau}\right\rangle$ is the radiation (number) state. The case of direct resonance energy transfer near a single mirror is depicted in Fig. 1. Here, the virtual photon can be created either at a donor or an acceptor. Therefore, with two virtual photon-matter interactions and the corresponding interaction Hamiltonian acting as a perturbation, the quantum amplitude can be calculated from the second term $(q=2)$ of the expansion given in Eq. (2),

$M_{F I}=\frac{\left\langle F\left|H_{\text {int }}\right| R_{1}^{1}\right\rangle\left\langle R_{1}^{1}\left|H_{\text {int }}\right| I\right\rangle}{E_{I}-E_{R_{1}^{1}}}+\frac{\left\langle F\left|H_{\text {int }}\right| R_{1}^{2}\right\rangle\left\langle R_{1}^{2}\left|H_{\text {int }}\right| I\right\rangle}{E_{I}-E_{R_{1}^{2}}}$,

where $I$ and $F$ are the initial and final states, respectively, and $R$ denotes the intermediate state.

\section{B. Mirror-assisted RET}

It is at this stage that the effect of including the mirror comes into play. We shall assume that the mirror is located at $z$ $=0$ and perpendicular to the $z$ axis. The electric field operator in the $z>0$ region will be quantized as ${ }^{29,45}$

$$
\begin{aligned}
\mathbf{E}_{\left(m, m^{\prime}\right)}\left(\mathbf{R}_{\xi}\right)= & \sum_{\mathbf{p}, \lambda}\left(\frac{\hbar c p}{2 V \epsilon_{0}}\right)^{1 / 2}\left[a^{(\lambda)}(\mathbf{p}) E_{\left(m, m^{\prime}\right)}\left(\mathbf{R}_{\xi}\right)\right. \\
& \left.-a^{\dagger(\lambda)}(\mathbf{p}) E_{\left(m, m^{\prime}\right)}^{*}\left(\mathbf{R}_{\xi}\right)\right],
\end{aligned}
$$

where $V$ is an arbitrary quantization volume and $a^{(\lambda)}(\mathbf{p})$ and $a^{\dagger(\lambda)}(\mathbf{p})$ are the familiar annihilation and creation operators that, respectively, modify the number of photons of modes; $m=\mathbf{p}, \lambda$ and $m^{\prime}=\mathbf{p}^{\prime}, \lambda . \mathbf{p}, \mathbf{p}^{\prime}$ are the incident and reflected wave vectors, respectively, and $\lambda$ is the polarization. Within each pair of braces, the paired terms effectively represent field 


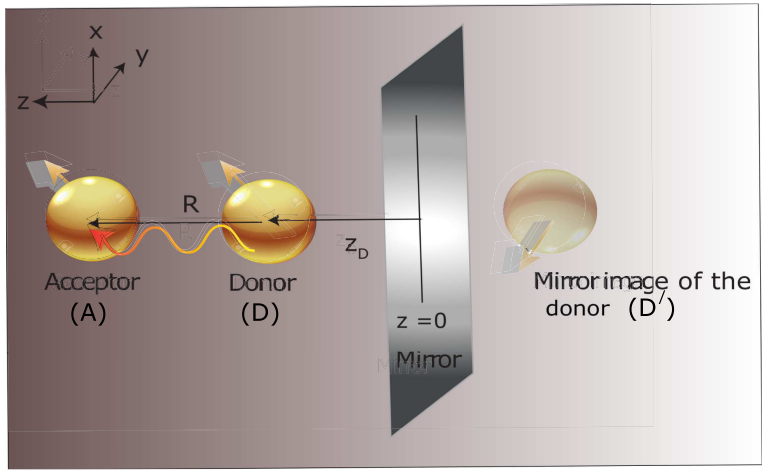

(a)

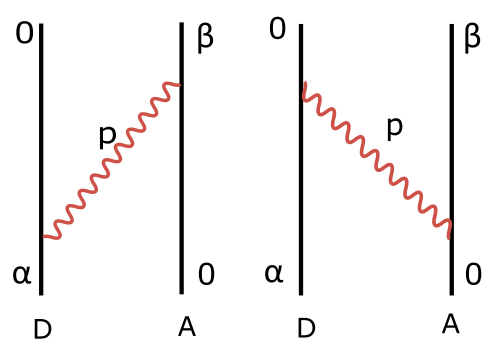

(b) expansions for the two separate half-spaces delineated by the mirror.

Therefore, the interaction matrix element can be derived from Eq. (6),

$$
\begin{aligned}
\left\langle R_{1}^{1}\left|H_{i n t}\right| I\right\rangle= & \left\langle 1(\mathbf{p}, \lambda) ; D^{0} A^{0}\right|-\boldsymbol{\mu}(D) . \mathbf{E}_{\left(m, m^{\prime}\right)}\left(\mathbf{R}_{D}\right) \\
& -\boldsymbol{\mu}(A) \cdot \mathbf{E}_{\left(m, m^{\prime}\right)}\left(\mathbf{R}_{A}\right)\left|D^{\alpha} A^{0} ; 0\right\rangle \\
= & \mathrm{i} \sum_{m}\left(\frac{\hbar c p}{2 V \epsilon_{0}}\right)^{1 / 2} \mu_{i}^{0 \alpha}(D) E_{\left(m, m^{\prime}\right) i}^{*}\left(\mathbf{R}_{D}\right),
\end{aligned}
$$

where $i, j$ are the Cartesian coordinates,

$$
\begin{aligned}
\left\langle F\left|H_{i n t}\right| R_{1}^{1}\right\rangle & =-\mathrm{i} \sum_{m, m^{\prime}}\left(\frac{\hbar c p}{2 V \epsilon_{0}}\right)^{1 / 2} \mu_{j}^{\beta 0}(A) E_{\left(m, m^{\prime}\right) j}\left(\mathbf{R}_{A}\right), \\
\left\langle R_{1}^{2}\left|H_{\text {int }}\right| I\right\rangle & =\mathrm{i} \sum_{m, m^{\prime}}\left(\frac{\hbar c p}{2 V \epsilon_{0}}\right)^{1 / 2} \mu_{i}^{\beta 0}(A) E_{\left(m, m^{\prime}\right) i}^{*}\left(\mathbf{R}_{A}\right), \\
\left\langle F\left|H_{\text {int }}\right| R_{1}^{2}\right\rangle & =-\mathrm{i} \sum_{m, m^{\prime}}\left(\frac{\hbar c p}{2 V \epsilon_{0}}\right)^{1 / 2} \mu_{j}^{0 \alpha}(D) E_{\left(m, m^{\prime}\right) j}\left(\mathbf{R}_{D}\right) .
\end{aligned}
$$

By the application of Eqs. (8) and (9) in Eq. (6) gives the general formula for the quantum amplitude for RET,

$$
\begin{aligned}
M_{F I}= & \frac{\mu_{i}^{0 \alpha}(D) \mu_{j}^{\beta 0}(A)}{2 V \epsilon_{0}} \sum_{m, m^{\prime}} \frac{E_{\left(m, m^{\prime}\right) i}^{*}\left(\mathbf{R}_{D}\right) E_{\left(m, m^{\prime}\right) j}\left(\mathbf{R}_{A}\right) p}{k-p} \\
& -\frac{E_{\left(m, m^{\prime}\right) i}\left(\mathbf{R}_{D}\right) E_{\left(m, m^{\prime}\right) j}^{*}\left(\mathbf{R}_{A}\right) p}{k+p} .
\end{aligned}
$$

In the above expression, a concise notation for the transition dipole moments is introduced, e.g., $\mu^{0 \alpha}(D) \equiv\left\langle D^{0}|\mu(D)| D^{\alpha}\right\rangle$, and $p$ is the corresponding photon wave number that need not be equal to $k$.

\section{Remarks on the proposed QED model}

It is important to highlight the fact that in our model, the light-matter interactions between the donor and acceptor have been considered within a perturbative quantum electrodynamics framework. ${ }^{29}$ Imposing quantum conditions on the classical electromagnetic (EM) field is the key step in quantum electrodynamics. In many instances, this is clearly seen by the appearance of reduced Planck's constant, $\hbar$, in expressions. Nevertheless, the final expression of the coupling matrix element, which is derived from the second order perturbation, is free from $\hbar$. This is because the $\hbar$ terms in the coupling matrix element cancel each other out. ${ }^{16}$ Therefore, this should not be misinterpreted as a classical result. ${ }^{46}$ The final expression for the resonance energy transfer essentially contains $\hbar$ from Fermi's golden rule in Eq. (1). ${ }^{13}$

\section{MIRROR ASSISTED RESONANCE ENERGY TRANSFER IN NANOSTRUCTURES}

It is fascinating to investigate the near field energy transfer based on QED under man-made alterations, enforced by confinement to regions bounded by surfaces like mirrors. Such effects are now becoming important in practice, primarily in quantum-optics experiments on particles in cavities that are bounded in various degrees. ${ }^{33,47,48}$ Therefore, we consider a system composed of two nanoparticles separated by a distance $R$ (center-to-center separation) and a single mirror $(z=0)$ as illustrated in Fig. 1. In this section, we analytically explore the effects on the process of energy transfer due to a mirror located in the vicinity of donor and acceptor nanostructures.

Now, let us consider donor and acceptor nanoparticles in the $x \mathrm{z}$ plane, and the coordinates are, respectively, $\mathbf{R}_{D}=(0,0$, $\left.z_{D}\right)$ and $\mathbf{R}_{A}=\left(R_{x}, 0, z_{D}+R\right) . R_{x}$ is the distance to the acceptor in the $x$ direction from the $z$ axis, and $R$ is the center-to-center separation distance between donor and acceptor particles. A single mirror in the $x, y$ plane is placed at $z=0$, as illustrated in Fig. $1(\mathrm{a}) ; z_{D}$ is the distance to the donor from the mirror in the $z$ direction. The corresponding Feynman diagrams are illustrated in Fig. 1(b).

\section{A. RET: Quantum dot to quantum dot in the vicinity of a mirror}

Here, a pair of QDs separated by distance $R$ interacting with a single mirror is considered. Thus, from Eq. (7), by considering electromagnetic waves in either side of the mirror, $E_{\left(m, m^{\prime}\right)}\left(\mathbf{R}_{\xi}\right)=\boldsymbol{e}^{(\lambda)}(\mathbf{p}) e^{\mathrm{i} \mathbf{p} \cdot \mathbf{R}_{\xi}}-\boldsymbol{e}^{(\lambda)}\left(\mathbf{p}^{\prime}\right) e^{i \mathbf{p}^{\prime} \cdot \mathbf{R}_{\xi}}$. Here, $\boldsymbol{e}^{(\lambda)}(\mathbf{p})$ is the polarization unit vector $\left[\overline{\boldsymbol{e}}^{(\lambda)}(\mathbf{p})\right.$ being its complex conjugate], now owing to the spherical symmetry,

$$
\begin{aligned}
\mathbf{p} & =p(\sin (\theta) \cos (\phi), \sin (\theta) \sin (\phi), \cos (\theta)), \\
\mathbf{p}^{\prime} & =p(\sin (\theta) \cos (\phi), \sin (\theta) \sin (\phi),-\cos (\theta)) .
\end{aligned}
$$

The wave-vector and polarization summations can be evaluated by following the standard techniques of Craig and Thirunamachandran ${ }^{13}$ [i.e., choosing the orthogonal frame set 
$\boldsymbol{e}^{(1)}(\mathbf{p}), \boldsymbol{e}^{(2)}(\mathbf{p})$ and $\mathbf{p}$ as the independent frame gives $\left.\boldsymbol{e}_{i}^{(1)}(\mathbf{p}) \overline{\boldsymbol{e}}_{j}^{(1)}(\mathbf{p})+\boldsymbol{e}_{i}^{(2)}(\mathbf{p}) \overline{\boldsymbol{e}}_{j}^{(2)}(\mathbf{p})+\hat{p}_{i} \hat{p}_{j}=\delta_{i j}\right]$. Finally, extending the boundaries of the quantization volume, each point in the $\mathbf{p}$-space represents a realizable $\mathbf{p}$-vector and the wave vector converts to an integral as $V \longrightarrow \infty$,

$$
\lim _{V \rightarrow \infty} \frac{1}{V} \sum_{\mathbf{p}} \equiv \int \frac{d^{3} \mathbf{p}}{(2 \pi)^{3}}
$$

Now converting to the spherical coordinates,

$$
\sum_{p} \Rightarrow \frac{V}{4 \pi^{3}} \int_{0}^{\infty} p^{2} d p \int_{0}^{\pi / 2} \sin (\theta) d \theta \int_{0}^{2 \pi} d \phi .
$$

Next, directly substituting into Eq. (10) and converting the discrete summation over the virtual photon wave vector give $^{18,29}$

$$
\begin{aligned}
M(Q D)= & \frac{1}{16 \pi^{3} \epsilon_{0}} \int_{0}^{\infty} d p \int_{0}^{\pi / 2} d \theta \int_{0}^{2 \pi} d \phi p^{3} \sin (\theta) \sum_{\lambda=1,2} \frac{\mu_{i}^{0 \alpha}(D) \mu_{j}^{\beta 0}(A)}{\left(k^{2}-p^{2}\right)}\left[\boldsymbol { e } _ { i } ^ { ( \lambda ) } ( \mathbf { p } ) \boldsymbol { e } _ { j } ^ { ( \lambda ) } ( \mathbf { p } ) \left\{k \left(e^{\mathrm{i} p\left(R_{x} \sin (\theta) \cos (\phi)+R \cos (\theta)\right)}\right.\right.\right. \\
& \left.\left.-e^{-\mathrm{i} p\left(R_{x} \sin (\theta) \cos (\phi)+R \cos (\theta)\right)}\right)+p\left(e^{\mathrm{i} p\left(R_{x} \sin (\theta) \cos (\phi)+R \cos (\theta)\right)}-e^{-\mathrm{i} p\left(R_{x} \sin (\theta) \cos (\phi)+R \cos (\theta)\right)}\right)\right\} \\
& +\boldsymbol{e}_{i}^{(\lambda)}\left(\mathbf{p}^{\prime}\right) \boldsymbol{e}_{j}^{(\lambda)}\left(\mathbf{p}^{\prime}\right)\left\{k\left(e^{\mathrm{i} p\left(R_{x} \sin (\theta) \cos (\phi)-R \cos (\theta)\right)}-e^{-\mathrm{i} p\left(R_{x} \sin (\theta) \cos (\phi)-R \cos (\theta)\right)}\right)+p\left(e^{\mathrm{i} p\left(R_{x} \sin (\theta) \cos (\phi)-R \cos (\theta)\right)}\right.\right. \\
& \left.\left.-e^{-\mathrm{i} p\left(R_{x} \sin (\theta) \cos (\phi)-R \cos (\theta)\right)}\right)\right\}+\boldsymbol{e}_{i}^{(\lambda)}(\mathbf{p}) \boldsymbol{e}_{j}^{(\lambda)}\left(\mathbf{p}^{\prime}\right)\left\{k \left(-e^{\mathrm{i} p\left(R_{x} \sin (\theta) \cos (\phi)-\left(R+2 z_{D}\right) \cos (\theta)\right)}\right.\right. \\
& \left.\left.+e^{-\mathrm{i} p\left(R_{x} \sin (\theta) \cos (\phi)-\left(R+2 z_{D}\right) \cos (\theta)\right)}\right)+p\left(-e^{\mathrm{i} p\left(R_{x} \sin (\theta) \cos (\phi)-\left(R+2 z_{D}\right) \cos (\theta)\right)}+e^{-\mathrm{i} p\left(R_{x} \sin (\theta) \cos (\phi)-\left(R+2 z_{D}\right) \cos (\theta)\right)}\right)\right\} \\
& +\boldsymbol{e}_{i}^{(\lambda)}\left(\mathbf{p}^{\prime}\right) \boldsymbol{e}_{j}^{(\lambda)}(\mathbf{p})\left\{k\left(-e^{\mathrm{i} p\left(R_{x} \sin (\theta) \cos (\phi)+\left(R+2 z_{D}\right) \cos (\theta)\right)}+e^{-\mathrm{i} p\left(R_{x} \sin (\theta) \cos (\phi)+\left(R+2 z_{D}\right) \cos (\theta)\right)}\right)\right. \\
& \left.\left.+p\left(-e^{\mathrm{i} p\left(R_{x} \sin (\theta) \cos (\phi)+\left(R+2 z_{D}\right) \cos (\theta)\right)}-e^{-\mathrm{i} p\left(R_{x} \sin (\theta) \cos (\phi)+\left(R+2 z_{D}\right) \cos (\theta)\right)}\right)\right\}\right]
\end{aligned}
$$

Performing the contour integration and applying the residue theorem, when $R_{x}=0$, yield

$$
\begin{aligned}
M(Q D)= & \frac{\mu_{i}^{0 \alpha}(D) \mu_{j}^{\beta 0}(A)}{4 \pi \epsilon_{0}}\left[-k^{2}\left(\delta_{i j}-\hat{R}_{i} \hat{R}_{j}\right)\left(\frac{\cos (k R)}{R}-\frac{\cos \left(k\left(R+2 z_{D}\right)\right)}{\left(R+2 z_{D}\right)}\right)+k\left(\delta_{i j}-3 \hat{R}_{i} \hat{R}_{j}\right)\left(\frac{\sin (k R)}{R^{2}}-\frac{\sin \left(k\left(R+2 z_{D}\right)\right)}{\left(R+2 z_{D}\right)^{2}}\right)\right. \\
& +\left(\delta_{i j}-3 \hat{R}_{i} \hat{R}_{j}\right)\left(\frac{\cos (k R)}{R^{3}}-\frac{\cos \left(k\left(R+2 z_{D}\right)\right)}{\left(R+2 z_{D}\right)^{3}}\right)+\mathrm{i}\left\{-k^{2}\left(\delta_{i j}-\hat{R}_{i} \hat{R}_{j}\right)\left(\frac{\sin (k R)}{R}-\frac{\sin \left(k\left(R+2 z_{D}\right)\right)}{\left(R+2 z_{D}\right)}\right)\right. \\
& \left.\left.-k\left(\delta_{i j}-3 \hat{R}_{i} \hat{R}_{j}\right)\left(\frac{\cos (k R)}{R^{2}}-\frac{\cos \left(k\left(R+2 z_{D}\right)\right)}{\left(R+2 z_{D}\right)^{2}}\right)+\left(\delta_{i j}-3 \hat{R}_{i} \hat{R}_{j}\right)\left(\frac{\sin (k R)}{R^{3}}-\frac{\sin \left(k\left(R+2 z_{D}\right)\right)}{\left(R+2 z_{D}\right)^{3}}\right)\right\}\right] .
\end{aligned}
$$

On the examination of Eq. (16), it is evident that the third term (in both real and imaginary components) is dominant in the short-range $(k R \ll 1)$, and the first term is dominant in the long-range $(k R \gg 1)$. Moreover, it can be shown that the coupling matrix element is fully transverse with respect to $\boldsymbol{R}$ in the long-range, whilst for the shorter-range, it contains both transverse and longitudinal components. ${ }^{18}$

RET occurs in the near-zone region. Hence, we will explicitly focus on the short-range for both QD and NW cases.

Thus, by applying $k R \ll 1$ for the near-field zone, and if $\mu(D), \mu(A)$ are in the positive $x$ direction,

$$
\begin{aligned}
M_{x x}(Q D)= & \frac{\mu_{x}(D)^{0 \alpha} \mu_{x}^{\beta 0}(A)}{4 \pi \epsilon_{0}}\left[\left\{\frac{1}{R^{3}}-\frac{\cos \left(2 k z_{D}\right)}{\left(R+2 z_{D}\right)^{3}}\right\}\right. \\
& \left.-\mathrm{i}\left\{\frac{\sin \left(2 k z_{D}\right)}{\left(R+2 z_{D}\right)^{3}}\right\}\right] .
\end{aligned}
$$

Moreover, the magnitude of the coupling matrix element can be expressed as

$$
\begin{aligned}
& \left|M_{x x}(Q D)\right|=\frac{\left|\mu_{x}(D)^{0 \alpha}\right|\left|\mu_{x}^{\beta 0}(A)\right|}{4 \pi \epsilon_{0}}
\end{aligned}
$$

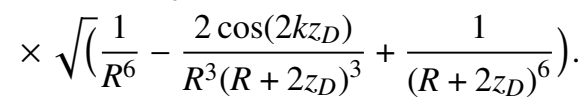

The plots of the normalized coupling matrix element are shown in Fig. 2. In the development of the plots for Sec. III, the following values were used for dipole moments: ${ }^{49}\left|\mu^{0 \alpha}(D)\right|$ $=\left|\mu^{\beta 0}(A)\right|=5 \times 10^{-30} \mathrm{C} \mathrm{m}$. Figure 2(b) shows the normalized RDDI (resonant dipole-dipole interaction) for various values of the separation distance of the mirror and the donor quantum $\operatorname{dot}\left(z_{D}\right)$. For the smaller separation distances, the RDDI with the presence of a mirror is lower than the RDDI of the mirrorless case.

\section{1. $Q D-Q D:$ Mirror-less case}

When the mirror-donor separation distance is large $\left(z_{D} \rightarrow \infty\right)$, relative $\mathrm{RDDI}\left(M / M_{0}\right)$ reaches 1 , reducing the result of Eq. (16) to the no-mirror expression given as

$$
\begin{aligned}
\lim _{z_{D} \rightarrow \infty} M(Q D)= & \frac{\mu_{i}^{0 \alpha}(D) \mu_{j}^{\beta 0}(A)}{4 \pi \epsilon_{0} R^{3}}\left\{\left(\delta_{i j}-3 \hat{R}_{i} \hat{R}_{j}\right)(\cos (k R)\right. \\
& \left.+k R \sin (k R))-\left(\delta_{i j}-\hat{R}_{i} \hat{R}_{j}\right)\left(k^{2} R^{2} \cos (k R)\right)\right\} \\
& -i\left\{\left(\delta_{i j}-3 \hat{R}_{i} \hat{R}_{j}\right)(\sin (k R)-k R \cos (k R))\right. \\
& \left.-\left(\delta_{i j}-\hat{R}_{i} \hat{R}_{j}\right)\left(k^{2} R^{2} \sin (k R)\right)\right\}
\end{aligned}
$$

This is exactly the same result obtained for direct coupling between two quantum dots when there is no mirror placed 


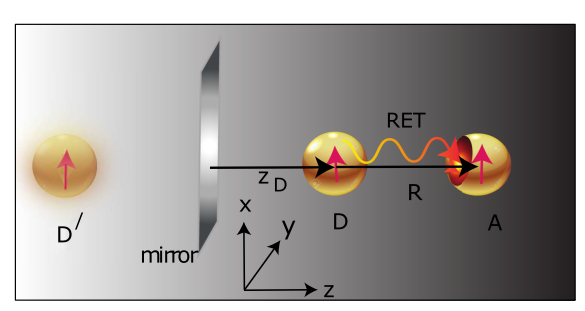

(a)

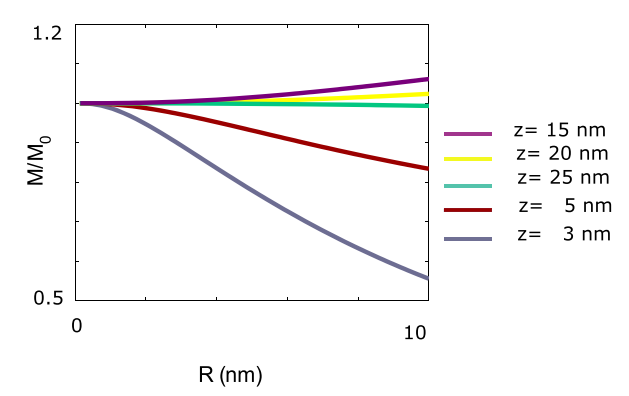

(c)

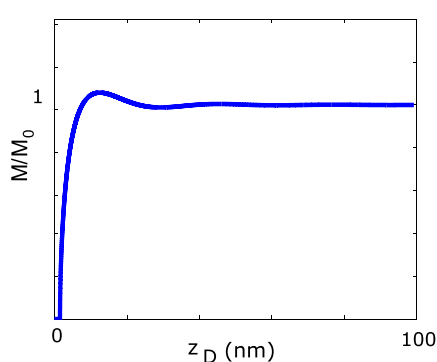

(b)

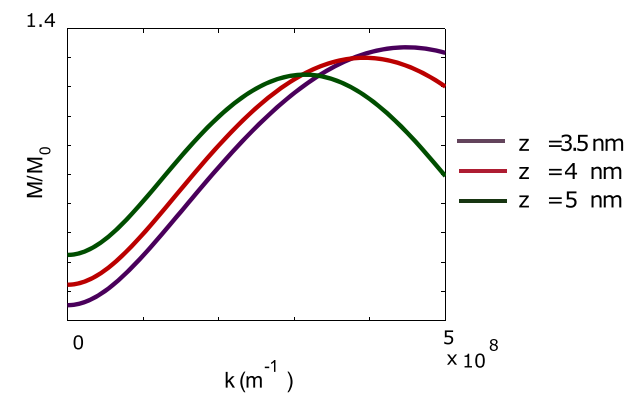

(d)
FIG. 2. Normalized RDDI strengths for two quantum dots parallel to the mirror: (a) schematic for the coupling of QD-QD near a mirror, (b) the normalized coupling matrix element $|M| /\left|M_{0}\right|$ as a function of the distance from the donor quantum dot, $D$, to the mirror, $z_{D}$, where the particle separation $R=10$ $\mathrm{nm}$, (c) the normalized coupling matrix element $|M| /\left|M_{0}\right|$ as a function of the donor-acceptor separation distance, $R$, for five different $z_{D}(3 \mathrm{~nm}, 5 \mathrm{~nm}, 15$ $\mathrm{nm}, 20 \mathrm{~nm}$, and $25 \mathrm{~nm}$ ), and (d) normalized RDDI strengths as a function of the wave number corresponding to the resonant atomic transition frequency for three different cases $\left(z_{D}=3.5 \mathrm{~nm}, 4 \mathrm{~nm}\right.$, and $5 \mathrm{~nm}$ ). nearby. ${ }^{21,25,50}$ Therefore it can be deduced from the above results that by applying the limits $\left(z_{D} \rightarrow \infty\right)$, we can successfully simplify the mirror-assisted RET to the no-mirror case (direct RET between two QDs).

Figures 2(c) and 2(d) depict the variation of normalized RDDI strengths with respect to the donor-acceptor separation distance $(R)$ and photon wave number corresponding to the atomic transition frequency $(k)$ for various $z_{D}$ values. As shown in Fig. 2(c), for larger $z_{D}$ values, an enhancement in the coupling matrix element compared with the mirror-less RDDI can be observed. However, a reduction in the coupling between donor-acceptor particles can be seen for smaller $z_{D}$ values. Interestingly, regardless of the position of the mirror, when the donor and acceptor are located in very close proximity, the influence of the mirror becomes negligible.

Moreover, the normalized coupling matrix element oscillates with the wave number, as illustrated in Fig. 2(d).
Furthermore, when the mirror is moved closer to the quantum dots, higher energy enhancement or more deterioration can be achieved depending on the suitably chosen $k$ value.

Substitution of Eq. (17) into the Fermi golden rule expression yields

$$
\begin{aligned}
\Gamma_{\text {trans }}(Q D)= & \frac{\left|\mu_{x}(D)^{0 \alpha}\right|^{2}\left|\mu_{x}^{\beta 0}(A)\right|^{2} \rho}{8 \pi \hbar \epsilon_{0}^{2}} \\
& \times\left(\frac{1}{R^{6}}-\frac{2 \cos \left(2 k z_{D}\right)}{R^{3}\left(R+2 z_{D}\right)^{3}}+\frac{1}{\left(R+2 z_{D}\right)^{6}}\right) .
\end{aligned}
$$

We illustrate the normalized energy transfer rates for three different energy shifting cases as shown in Fig. 3. Figure 3(a) shows the RET rate enhancement factor as a percentage with respect to the donor-acceptor separation distance, for three mirror to donor distances $\left(z_{D}=8 \mathrm{~nm}, 15 \mathrm{~nm}\right.$, and $\left.20 \mathrm{~nm}\right)$. It can be observed that the normalized energy transfer rate
Energy transfer enhancement

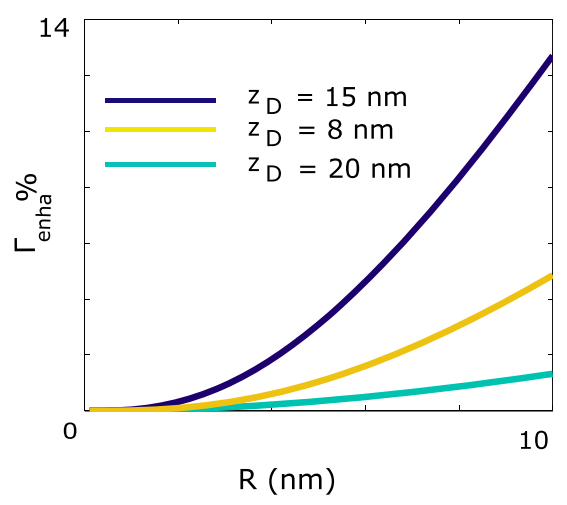

(a)

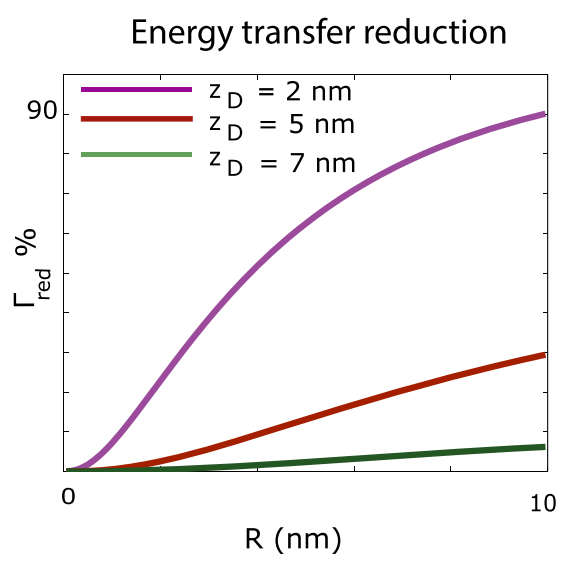

(b)

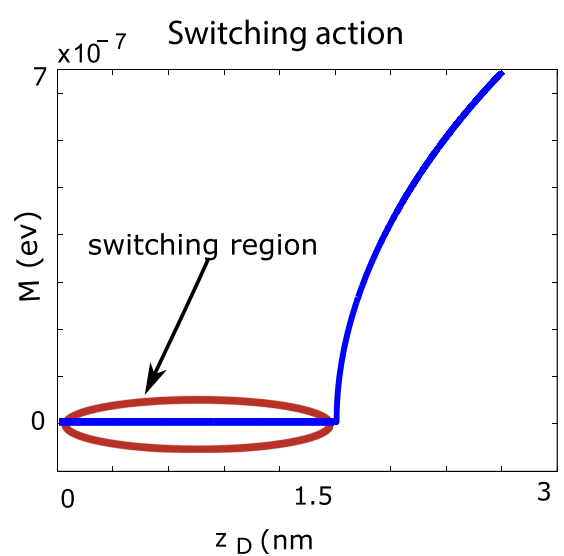

(c)

FIG. 3. Normalized energy transfer rates for three different energy shifting cases: (a) RET rate enhancement factor (as a percentage) in QDs as a function of the donor-acceptor separation distance, for three mirror to donor distances $\left(z_{D}=8 \mathrm{~nm}, 15 \mathrm{~nm}\right.$, and $20 \mathrm{~nm}$ ), (b) RET rate deterioration factor (as a percentage) in QDs as a function of the donor-acceptor separation distance, for three different mirror to donor distances $\left(z_{D}=2 \mathrm{~nm}, 5 \mathrm{~nm}\right.$, and $\left.7 \mathrm{~nm}\right)$, and (c) switching action: the coupling matrix element as a function of $z_{D}$. 
gradually increases with $R$. Furthermore, the energy transfer can also be diminished for certain mirror-particle separation distances as depicted in Fig. 3(b).

Moreover, the RET can be completely "cutoff" in the region highlighted in Fig. 3(c) owing to the fact that the electric field component [see Eq. (7)] is modified by the light reflected from the mirror surface. In Fig. 3(c), the center-center separation distance of donor and acceptor QDs and the wave number are $R=10 \mathrm{~nm}$ and $k=1 \times 10^{8} \mathrm{~m}^{-1}$, respectively. The switching region varies according to the values of these parameters. This can be understood by careful examination of Eq. (18). Here, when $z_{D}$ is small, one of the additional terms introduced by the reflected waves from the mirror $\left(\frac{2 \cos \left(2 k z_{D}\right)}{R^{3}\left(R+2 z_{D}\right)^{3}}\right)$ becomes more dominant and makes the coupling matrix element 0 or negligible. When $z_{D}$ increases, a sudden upturn of the RDDI can be observed. This is due to the high influence of smaller $z_{D}$ values on the RDDI, for a suitably oriented QD pair. This high impact of $z_{D}$ on the RDDI gradually decreases to 0 with increasing $z_{D}$ [applying $z_{D} \rightarrow \infty$ limits on Eq. (17)]. This drastic upturn opens up potential applications for highly sensitive positioning tools. $^{51-53}$

\section{B. RET: Nanowire to nanowire in the vicinity of a mirror}

In this section, the process of RET in a system consisting of idealized 2D parallel nanowires of length $L$ is considered. Owing to the cylindrical symmetry of NWs, it is convenient to model EM waves using a Hankel function of order $n .^{25,54,55}$

In a similar manner to the previous case in Sec. III A, if $\mu(D), \mu(A)$ are oriented in the positive $x$ direction and two nanowires are located along the $z$ axis $\left(R_{x}=0\right)$ as shown in Fig. 4(a), then we have

$$
\begin{aligned}
M_{x x}(N W)= & \frac{\mu_{x}^{0 \alpha}(D) \mu_{x}^{\beta 0}(A) k^{2}}{4 L \epsilon_{0}}\left\{Y_{0}(k R)-Y_{0}\left(k R+2 k z_{D}\right)\right. \\
& \left.-\mathrm{i}\left(J_{0}(k R)-J_{0}\left(k R+2 k z_{D}\right)\right)\right\}
\end{aligned}
$$

Similarly, the magnitude of the quantum amplitude in the positive $x$ direction can be expressed as follows:

$$
\begin{aligned}
\left|M_{x x}(N W)\right|= & \frac{\left|\mu_{x}^{0 \alpha}(D)\right|\left|\mu_{x}^{\beta 0}(A)\right| k^{2}}{4 L \epsilon_{0}} \sqrt{\left\{Y_{0}^{2}(k R)+J_{0}^{2}(k R)\right.} \\
& +Y_{0}^{2}\left(k R+2 k z_{D}\right)+J_{0}^{2}\left(k R+2 k z_{D}\right) \\
& -2 Y_{0}(k R) Y_{0}\left(k R+2 k z_{D}\right) \\
& \left.-2 J_{0}(k R) J_{0}\left(k R+2 k z_{D}\right)\right\}
\end{aligned}
$$

We depict the normalized coupling matrix element for NW-NW in the vicinity of a mirror as shown in Fig. 4. Figure 4(b) shows the normalized RDDI for various values of the separation distance $\left(z_{D}\right)$ of the mirror and the donor NW. For the smaller separation distances, the RDDI with the mirror in the vicinity is lower than the RDDI of the mirror-less case. The normalized coupling matrix element fluctuates around the value of 1 and gradually decreases with $z_{D}$.

\section{NW-NW: Mirror-less case}

Similar to the QD case, for higher mirror-donor separation distances $\left(z_{D} \rightarrow \infty\right)$, relative RDDI reaches 1 , reducing the result of Eq. (21) to the mirror-less expression given as

$$
\lim _{z_{D} \rightarrow \infty} M_{x x}(N W)=\frac{\mu_{x}^{0 \alpha}(D) \mu_{x}^{\beta 0}(A) k^{2}}{4 L \epsilon_{0}}\left\{Y_{0}(k R)-\mathrm{i}\left(J_{0}(k R)\right)\right\} .
$$

The above expression can also be obtained by deriving the direct coupling between $\mathrm{NW}$ to $\mathrm{NW}$, from the second order perturbation theory. ${ }^{25,55}$ Therefore, by imposing the limits $\left(z_{D} \rightarrow \infty\right)$, we can successfully simplify the mirror-assisted RET to the no-mirror case (direct RET).

The magnitude of the quantum amplitude in the absence of the mirror becomes

$$
\left|M_{0}(N W)\right|=\frac{\left|\mu^{0 \alpha}(D)\right|\left|\mu^{\beta 0}(A)\right| k^{2}}{4 L \epsilon_{0}} \sqrt{\left\{Y_{0}^{2}(k R)+J_{0}^{2}(k R)\right\} .}
$$

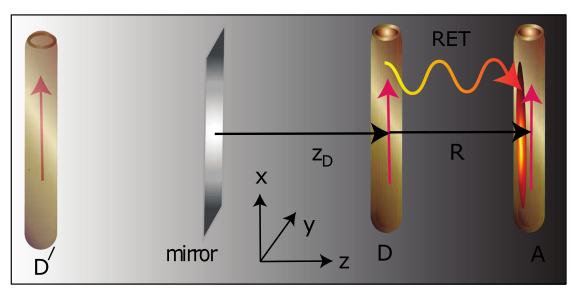

(a)

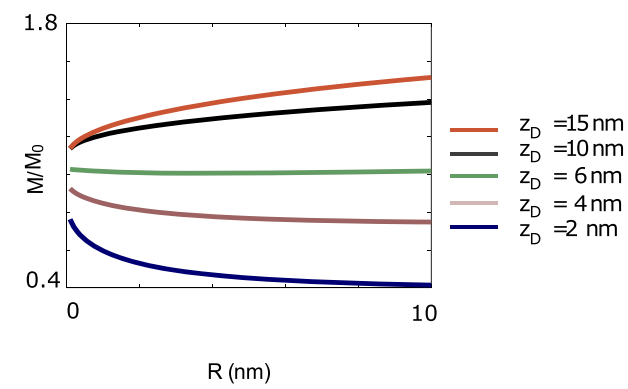

(c)

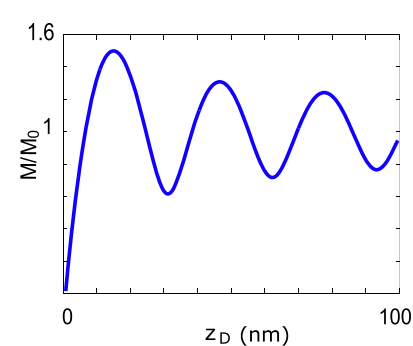

(b)

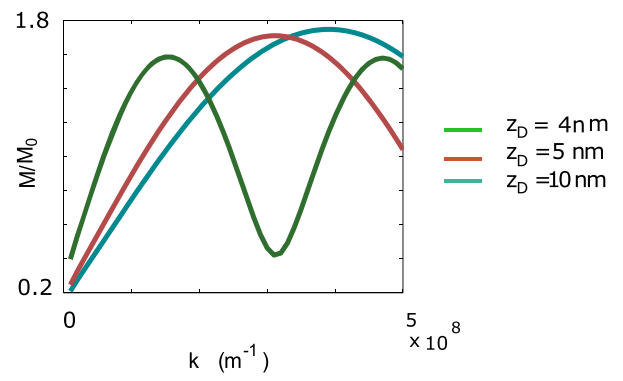

(d)
FIG. 4. Normalized RDDI strengths for two nanowires parallel to the mirror: (a) schematic for the coupling of NW-NW in the vicinity of a mirror, (b) the normalized coupling matrix element $|M| /\left|M_{0}\right|$ as a function of the distance from the donor nanowire, $D$, to the mirror, $z_{D}$, where the particle separation $R$ $=10 \mathrm{~nm}$, (c) the normalized coupling matrix element $|M| /\left|M_{0}\right|$ as a function of the donor-acceptor separation distance, $R$, for five different values of $z_{D}(2 \mathrm{~nm}$, $4 \mathrm{~nm}, 6 \mathrm{~nm}, 10 \mathrm{~nm}$, and $15 \mathrm{~nm}$ ), and (d) normalized RDDI strengths as a function of the wave number corresponding to the resonant atomic transition frequency for three different cases $\left(z_{D}\right.$ $=4 \mathrm{~nm}, 5 \mathrm{~nm}$, and $10 \mathrm{~nm}$ ). 
As expected, owing to the photon behaviour in the cylindrical symmetry and the modified electric field component due to the mirror surface, the coupling between two nanowires modified to Eq. (22) from Eq. (24).

Furthermore, Figs. 4(c) and 4(d) show the variation of normalized RDDI strengths with respect to $R$ and photon wave number corresponding to the atomic transition frequency $(k)$ for various $z_{D}$ values. As shown in Fig. 4(c), for larger $z_{D}$ values, an enhancement in the coupling matrix element compared with the mirror-less RDDI can be observed. However, a reduction in the coupling between donor-acceptor particles can be seen for smaller $z_{D}$ values. Similar to the case of QDs, regardless of the position of the mirror, when the donor and acceptor are located in very close proximity, the influence of the mirror becomes negligible, converging the normalized factor to 1 . In addition, unlike in the variation of the normalized matrix element with $k$ in QDs, same amount of enhancement and reduction of the quantum amplitude can be observed for different $z_{D}$ values in NWs due to the behaviour of the virtual photon propagation in a 2D realm [see Fig. 4(d)]. To determine the rate of transfer, Fermi's rule given in Eq. (1) can be used,

$$
\begin{gathered}
\Gamma_{\text {trans }}(N W)=\frac{\left|\mu_{x}^{0 \alpha}(D)\right|^{2}\left|\mu_{x}^{\beta 0}(A)\right|^{2} k^{4} \pi \rho}{8 L^{2} \hbar \epsilon_{0}^{2}}\left\{Y_{0}^{2}(k R)+J_{0}^{2}(k R)+Y_{0}^{2}\left(k R+2 k z_{D}\right)+J_{0}^{2}\left(k R+2 k z_{D}\right)\right. \\
\left.-2 Y_{0}(k R) Y_{0}\left(k R+2 k z_{D}\right)-2 J_{0}(k R) J_{0}\left(k R+2 k z_{D}\right)\right\}, \\
\frac{\Gamma_{\text {trans }}(N W)}{\Gamma_{\text {trans }, 0}(N W)}=\frac{Y_{0}^{2}(k R)+J_{0}^{2}(k R)+Y_{0}^{2}\left(k R+2 k z_{D}\right)+J_{0}^{2}\left(k R+2 k z_{D}\right)-2 Y_{0}(k R) Y_{0}\left(k R+2 k z_{D}\right)-2 J_{0}(k R) J_{0}\left(k R+2 k z_{D}\right)}{Y_{0}^{2}(k R)+J_{0}^{2}(k R)} .
\end{gathered}
$$

The plots of rate enhancement and reduction factors for Eq. (26) are shown in Figs. 5(a) and 5(b) for various values of the D-A distance, $R$. These figures exhibit similar patterns to those observed for QDs in Sec. III A. In fact, 130\% increment of the energy transfer can be gained when the mirror is placed $15 \mathrm{~nm}$ away from the donor NW. Additionally, up to $80 \%$ transfer inhibition can be obtained when $z_{D}=2 \mathrm{~nm}$.

\section{Design guidelines for mirror-assisted RET}

In Secs. III A and III B, we have developed a fundamental theory for describing the mirror-assisted resonance energy transfer in dimensionally constrained nanostructures (QDs and NWs) within the framework of quantum electrodynamics. We have also shown that transfer rates can be robustly enhanced or suppressed by positioning a mirror near a donor-acceptor pair under favourable conditions.

Therefore, in this section, we will elaborate design guidelines for mirror-assisted RET with suitable model parameters. The following values were used for generation of plots: ${ }^{49}\left|\mu^{0 \alpha}(D)\right|=\left|\mu^{\beta 0}(A)\right|=5 \times 10^{-30} \mathrm{Cm}$ and $k=1$ $\times 10^{8} \mathrm{~m}^{-1}$. Moreover for all the plots generated, we used the donor-acceptor separation distance of $R$ less than $10 \mathrm{~nm}$. However, we exclude the results for $R<1 \mathrm{~nm}$ as those signify the possibility of wave function overlap (Dexter zone). ${ }^{56} \mathrm{~A}$ detailed explanation about this region is given in Sec. IV.

1. RET enhancement: Higher RET rate enhancements can be achieved in NWs than in QDs when placed in the vicinity of an ideal mirror.

- Quantum dots: Fig. 3(a) illustrates the energy transfer enhancement with respect to the donoracceptor separation distance. Up to $13 \%$ enhancement can be obtained when $z_{D} \leq 15 \mathrm{~nm}$.

- Nanowires: Approximately $130 \%$ increment of the energy transfer can be gained when the mirror is placed $15 \mathrm{~nm}$ away from the donor NW. This is shown in Fig. 5(a).

2. RET suppression: The RET rate can be successfully inhibited up to a considerable extent in both QDs and NWs.
Energy transfer enhancement

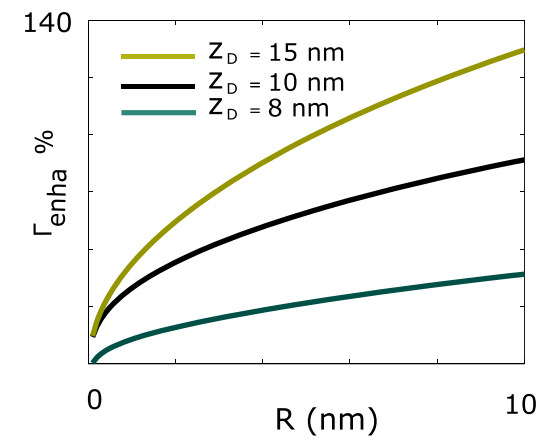

(a)

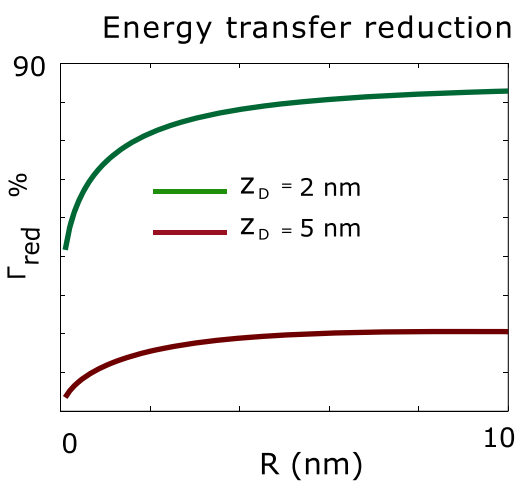

(b)
FIG. 5. Normalized energy transfer rates for two different energy shifting cases: (a) RET rate enhancement factor (as a percentage) in NWs as a function of donoracceptor separation distance, for three mirror to donor distances $\left(z_{D}=8 \mathrm{~nm}, 10 \mathrm{~nm}\right.$, and $15 \mathrm{~nm}$ ), (b) RET rate deterioration factor as a percentage in NWs as a function of donor-acceptor separation distance, for two different mirror to donor distances $\left(z_{D}=2 \mathrm{~nm}\right.$ and $\left.5 \mathrm{~nm}\right)$. 
- Quantum dots: When the donor-mirror separation distance is less than $7 \mathrm{~nm}$, the mirror-assisted RET rate starts going below $\Gamma_{0}$ (rate corresponding to the no-mirror case). This is because, when $z_{D}$ is small, one of the additional terms introduced by the reflected waves from the mirror $\left(\frac{2 \cos \left(2 k z_{D}\right)}{R^{3}\left(R+2 z_{D}\right)^{3}}\right)$ becomes more dominant making the coupling matrix element smaller than that of the no-mirror case. Thus, more than $90 \%$ of the rate reduction can be achieved when $z_{D}=2 \mathrm{~nm}$ as depicted in Fig. 3(b).

- Nanowires: Similar to the QDs, approximately when $z_{D}<6 \mathrm{~nm}$, RET rate suppression can be obtained. This is shown in Fig. 5(b) for three different $z_{D}$ values. Nearly $83 \%$ of the rate reduction can be observed when $z_{D}=2 \mathrm{~nm}$.

3. Switching action: This can be observed only in QDs, as illustrated in Fig. 3(c). A sudden up turn can be observed when the mirror is placed very close to the donor QD (i.e., $\sim 1.6 \mathrm{~nm}$, when $R=10 \mathrm{~nm}$ ).

\section{DISCUSSION}

A fundamental theory has been developed for describing the mirror assisted resonance energy transfer in dimensionally constrained nanostructures within the framework of QED. We have performed analytical derivations and plotted the coupling matrix elements and energy transfer rates. The results have demonstrated the effect of the mirror in the vicinity of two donor-acceptor nanostructures, according to the relative distances among each quantum object along with the variation of quantized EM modes; these are the factors that determine the controllability of the resonance energy transfer rate. This can be understood by the modification to the electric field ${ }^{29,45}$ given in Eq. (7), which contributes additional components to the coupling matrix elements as derived in Eqs. (10) and (21) for QDs and NWs, respectively. This quantum level element of interference allows a high degree of control of the RET rate (RET rate enhancement, suppression, and switching actions) between nanostructures.

Figure 2 characterizes the normalized RDDI factor of QD-QD as a function of the donor distance to the mirror, donoracceptor distance, and wave number (corresponding to the resonant atomic transition frequency, $\left.f_{r}=k c / 2 \pi\right)$. The range where the coupling between donor and acceptor QDs is effectively controlled by the distance to the mirror is $z_{D}<40 \mathrm{~nm}$. This range is set by the donor-acceptor distance of $20 \mathrm{~nm}$. However, if the donor-acceptor distance is set to the most typical RET separation distance in the order of $10 \mathrm{~nm}$, then the precise RET controlling range becomes $z_{D}<20 \mathrm{~nm}$. This agrees with the results of Ref. 38 .

Therefore, we have studied the coupling between donor and acceptor QDs as a function of $R$ and $k$, for various values of $z_{D}$, in the range of $z_{D}<20 \mathrm{~nm}$. Interestingly, when $8 \mathrm{~nm}$ $<z_{D}<20 \mathrm{~nm}$, the rate enhancement up to $13 \%$ can be achieved [see Figs. 2(c) and 3(a)]. In addition to this, when $z_{D}<8 \mathrm{~nm}$, the RET rate can be suppressed to a certain extent (90\%) as shown in Figs. 2(c) and 3(b). Furthermore, the drastic upturn shown in Fig. 3(c) opens up potential applications for highly sensitive positioning tools. ${ }^{51-53}$

In Sec. III B, similar results exhibit by the coupling between NW-NW near a single mirror. Nevertheless, in contrast to the QD case, a more than $120 \%$ increment in the energy transfer rate can be gained as shown in the plots of Fig. 5(a).

In contrast to the emitter configuration used in this paper, the work reported in Ref. 38 employed both parallel and perpendicular configurations of dipoles with respect to the mirror. Compared to the classical approach used in Ref. 38, the present paper formulated a full quantum electrodynamical treatment for mirror assisted energy migration with the aid of Feynman diagram methods. Interestingly, as in our case, the above article shows that the RET rate can be inhibited or enhanced by carefully positioning a mirror close to particles, typically a few $\mathrm{nm}$. In Ref. 38, higher enhancement can be observed compared with the rate inhibition for dipole emitters. Nevertheless, in our case, QDs exhibit higher percentages of inhibitions than enhancements, while NWs gain higher RET rate enhancements than inhibitions, when located near a mirror. Moreover, in the present article, we could achieve higher rate enhancements for NWs than QDs while both nanostructures show markedly rate inhibitions.

Note that values of $R<1 \mathrm{~nm}$ generally signify the possibility of wave function overlap. Here, another mechanism of energy migration occurs, called the Dexter mechanism. ${ }^{56}$ When the particles are almost touching each other and the interaction between them is high, there is a possibility that as the excited electron is transferred from the donor particle to the empty higher energy level of the acceptor particle, the electron in the ground state of the particle is simultaneously moved to fill the "electron hole" of the donor. The net physical result is the same as in the Förster transfer, but here the energy transfers follow an exponential rate formula. Nonetheless, in the kinds of nanoscale systems of widest interest, the donor and acceptor components are in general designedly separated by a distance sufficiently large to preclude any significant degree of wave function overlap. Therefore, in this article, we exclude the Dexter zone to focus specifically on the Förster range.

It is immediately clear that the far-zone and intermediatezone terms in Eq. (16) exhibit similar patterns to the near-zone results. However, it provides different coupling matrix element values due to the $k^{2}$ and $k$ terms in the numerator. In the longrange, the photons have field components that are transverse with respect to the displacement vector, opposed to the nearfield case, where both transverse and longitudinal components play vital roles in the process of energy migration. ${ }^{16}$ Nevertheless, the cases where the mirror positioned far-away from the donor-acceptor system in the far- and intermediate-zone provide results exactly similar to the respective no-mirror cases. This proves that our results are valid for all three domains: near-, intermediate-, and far-field.

\section{CONCLUSION AND OUTLOOK}

Tailoring the quantum level light-matter interaction with the nanophotonic environment provides accurate control over the resonance energy transfer between two nanostructure 
particles. Despite the wide applications of RET in nanosciences, using nanophotonics to control RET has remained a debated and complex challenge over many decades. Here we have demonstrated the exquisitely controlled energy transfer within a single donor-acceptor nanoparticle pair placed in close proximity of a mirror, at nanoscale distances comparable to the separation between the particles. A full quantum electrodynamical treatment for mirror-assisted energy migration has been formulated with the aid of Feynman diagrams and perturbational theory. ${ }^{57,58}$

It is interesting to note that other aspects of photophysics being markedly modified in the proximity of a mirror have also recently received attention in connection with chiral species and circular polarisations. ${ }^{59-62}$

Summarizing, the ensuing results demonstrated the possibility of altering the strength of the resonance energy transfer between two nanoparticles by careful engineering of the spacing and inclusion of an ideal quantum mirror in the vicinity. Therefore, the analysis demonstrates a mechanism to inhibit or markedly enhance the transfer of energy between discrete components, potentially in any multi-quantum dot/nanowire system, inviting surface and layer applications.

As future extensions of our work, it will be interesting to study the different orientations of the nanostructures with respect to the mirror. Moreover, a possible optical switching action with a laser beam impinging on the particle system represents scope for potential development of the theory.

In conclusion, we believe that our new insight into the control of the near-field resonance energy transfer offers considerable new scope to be exploited for accurately measuring molecular separations, for the manipulation of quantum information, and for much efficient bio-sensors, and holds potential for improved photovoltaics.

\section{ACKNOWLEDGMENTS}

The work of D.W. is supported by the Monash University Institute of Graduate Research. The work of M.P. is supported by the Australian Research Council, through its Discovery Grant No. DP140100883.

${ }^{1}$ M. Mohseni, P. Rebentrost, S. Lloyd, and A. Aspuru-Guzik, "Environmentassisted quantum walks in photosynthetic energy transfer," J. Chem. Phys. 129, 174106 (2008).

${ }^{2}$ P. Rebentrost, M. Mohseni, I. Kassal, S. Lloyd, and A. Aspuru-Guzik, "Environment-assisted quantum transport," New J. Phys. 11, 033003 (2009).

${ }^{3}$ R. van Grondelle and V. I. Novoderezhkin, "Energy transfer in photosynthesis: Experimental insights and quantitative models," Phys. Chem. Chem. Phys. 8, 793-807 (2006).

${ }^{4}$ T. Förster, "Zwischenmolekulare energiewanderung und fluoreszenz," Ann. Phys. 437, 55-75 (1948).

${ }^{5}$ M. Şener, J. Strümpfer, J. Hsin, D. Chandler, S. Scheuring, C. N. Hunter, and K. Schulten, "Förster energy transfer theory as reflected in the structures of photosynthetic light-harvesting systems," Chem. Phys. Chem. 12, 518-531 (2011).

${ }^{6}$ S. Buhbut, S. Itzhakov, E. Tauber, M. Shalom, I. Hod, T. Geiger, Y. Garini, D. Oron, and A. Zaban, "Built-in quantum dot antennas in dye-sensitized solar cells," ACS Nano 4, 1293-1298 (2010).

${ }^{7}$ G. D. Scholes, G. R. Fleming, A. Olaya-Castro, and R. van Grondelle, "Lessons from nature about solar light harvesting," Nat. Chem. 3, 763-774 (2011).
${ }^{8}$ D. Sikdar, W. Cheng, and M. Premaratne, "Optically resonant magnetoelectric cubic nanoantennas for ultra-directional light scattering," J. Appl. Phys. 117, 083101 (2015).

${ }^{9}$ I. L. Medintz, A. R. Clapp, H. Mattoussi, E. R. Goldman, B. Fisher, and J. M. Mauro, "Self-assembled nanoscale biosensors based on quantum dot FRET donors," Nat. Mater. 2, 630-638 (2003).

${ }^{10}$ K. F. Chou and A. M. Dennis, "Förster resonance energy transfer between quantum dot donors and quantum dot acceptors," Sensors 15, 13288-13325 (2015).

${ }^{11}$ B. Schuler, E. A. Lipman, and W. A. Eaton, "Probing the free-energy surface for protein folding with single-molecule fluorescence spectroscopy," Nature 419, 743-747 (2002).

${ }^{12}$ P. Carriba, G. Navarro, F. Ciruela, S. Ferré, V. Casadó, L. Agnati, A. Cortés, J. Mallol, K. Fuxe, E. I. Canela et al., "Detection of heteromerization of more than two proteins by sequential BRET-FRET," Nat. Methods 5, 727-733 (2008).

${ }^{13}$ D. P. Craig and T. Thirunamachandran, Molecular Quantum Electrodynamics (Dover, New York, 1998).

${ }^{14}$ J. S. Avery, "Resonance energy transfer and spontaneous photon emission," Proc. Phys. Soc. 88, 1 (1966).

${ }^{15}$ L. Gomberoff and E. A. Power, "The resonance transfer of excitation," Proc. Phys. Soc. 88, 281 (1966).

${ }^{16}$ D. L. Andrews, "A unified theory of radiative and radiationless molecular energy transfer," Chem. Phys. 135, 195-201 (1989).

${ }^{17}$ A. Salam, Molecular Quantum Electrodynamics: Long-Range Intermolecular Interactions (Wiley, Hoboken, NJ, 2010).

${ }^{18}$ D. L. Andrews and D. S. Bradshaw, "The role of virtual photons in nanoscale photonics," Ann. Phys. 526, 173-186 (2014).

${ }^{19}$ D. L. Andrews and A. A. Demidov, Resonance Energy Transfer (Wiley, 1999).

${ }^{20}$ D. L. Andrews and J. M. Leeder, "On the interactions between molecules in an off-resonant laser beam: Evaluating the response to energy migration and optically induced pair forces," J. Chem. Phys. 130, 034504 (2009).

${ }^{21}$ G. J. Daniels, R. D. Jenkins, D. S. Bradshaw, and D. L. Andrews, "Resonance energy transfer: The unified theory revisited," J. Chem. Phys. 119, 2264 2274 (2003).

${ }^{22}$ I. Medintz and N. Hildebrandt, FRET-Förster Resonance Energy Transfer: From Theory to Applications (John Wiley \& Sons, 2013).

${ }^{23}$ D. L. Andrews and J. Rodríguez, "Resonance energy transfer: Spectral overlap, efficiency, and direction," J. Chem. Phys. 127, 084509 (2007).

${ }^{24}$ A. Salam, "Mediation of resonance energy transfer by a third molecule," J. Chem. Phys. 136, 014509 (2012).

${ }^{25}$ D. Weeraddana, M. Premaratne, and D. L. Andrews, "Direct and thirdbody mediated resonance energy transfer in dimensionally constrained nanostructures," Phys. Rev. B 92, 035128 (2015).

${ }^{26}$ P. Allcock, R. D. Jenkins, and D. L. Andrews, "Laser-assisted resonanceenergy transfer," Phys. Rev. A 61, 023812 (2000).

${ }^{27}$ D. Weeraddana, M. Premaratne, S. D. Gunapala, and D. L. Andrews, "Quantum electrodynamical theory of high-efficiency excitation energy transfer in laser-driven nanostructure systems," Phys. Rev. B 94, 085133 (2016).

${ }^{28}$ D. Weeraddana, M. Premaratne, and D. L. Andrews, "Optical control of resonance energy transfer in quantum dot systems," in 2016 IEEE Nanotechnology Materials and Devices Conference (NMDC) (IEEE, 2016), pp. 1-2.

${ }^{29}$ T. Kobayashi, Q. Zheng, and T. Sekiguchi, "Resonance transfer of excitation for molecules between mirrors," Phys. Lett. A 199, 21-26 (1995).

${ }^{30}$ E. M. Purcell, "Spontaneous emission probabilities at radio frequencies," NATO ASI Series (Series B: Physics) 340, 839 (1995).

${ }^{31}$ P. R. Berman, Cavity Quantum Electrodynamics (Academic Press, Inc., Boston, MA, USA, 1994).

${ }^{32}$ M. D. LaCount and M. T. Lusk, "Electric dipole coupling in optical cavities and its implications for energy transfer, up-conversion, and pooling," Phys. Rev. A 93, 063811 (2016).

${ }^{33} \mathrm{P}$. Andrew and W. L. Barnes, "Förster energy transfer in an optical microcavity," Science 290, 785-788 (2000).

${ }^{34}$ C. Jayasekara, M. Premaratne, S. D. Gunapala, and M. I. Stockman, " $\mathrm{MoS}_{2}$ spaser," J. App. Phys. 119, 133101 (2016).

${ }^{35}$ A. O. Govorov, J. Lee, and N. A. Kotov, "Theory of plasmon-enhanced Förster energy transfer in optically excited semiconductor and metal nanoparticles," Phys. Rev. B 76, 125308 (2007).

${ }^{36}$ C. Rupasinghe, I. D. Rukhlenko, and M. Premaratne, "Spaser made of graphene and carbon nanotubes,” ACS Nano 8, 2431-2438 (2014). 
${ }^{37}$ M. Premaratne and M. I. Stockman, "Theory and technology of SPASERs," Adv. Opt. Photon. 9, 79-128 (2017).

${ }^{38} \mathrm{M}$. Wubs and W. L. Vos, "Förster resonance energy transfer rate in any dielectric nanophotonic medium with weak dispersion," New J. Phys. 18, 053037 (2016).

${ }^{39}$ R. Swathi and K. Sebastian, "Excitation energy transfer from a fluorophore to single-walled carbon nanotubes," J. Chem. Phys. 132, 104502 (2010).

${ }^{40}$ C. S. Kumarasinghe, M. Premaratne, S. D. Gunapala, and G. P. Agrawal, "Design of all-optical, hot-electron current-direction-switching device based on geometrical asymmetry," Sci. Rep. 6, 21470 (2016).

${ }^{41}$ M. Premaratne and G. P. Agrawal, Light Propagation in Gain Media (Cambridge University Press, 2011).

${ }^{42}$ A. Salam, "A general formula for the rate of resonant transfer of energy between two electric multipole moments of arbitrary order using molecular quantum electrodynamics," J. Chem. Phys. 122, 044112 (2005).

${ }^{43}$ L. Mandel and E. Wolf, Optical Coherence and Quantum Optics (Cambridge University Press, 1995).

${ }^{44}$ R. Woolley, "Gauge invariance in non-relativistic electrodynamics," Proc. R. Soc. A 456, 1803-1819 (2000).

${ }^{45}$ G. Barton, "Quantum electrodynamics of atoms between parallel mirrors," Phys. Scr. T21, 11 (1988).

${ }^{46}$ D. Andrews, D. Craig, and T. Thirunamachandran, "Molecular quantum electrodynamics in chemical physics," Int. Rev. Phys. Chem. 8, 339-383 (1989).

${ }^{47}$ R. Chikkaraddy, B. de Nijs, F. Benz, S. J. Barrow, O. A. Scherman, E. Rosta, A. Demetriadou, P. Fox, O. Hess, and J. J. Baumberg, "Single-molecule strong coupling at room temperature in plasmonic nanocavities," Nature 535, 127 (2016).

${ }^{48}$ M. Steiner, F. Schleifenbaum, C. Stupperich, A. Virgilio Failla, A. Hartschuh, and A. J. Meixner, "Microcavity-controlled single-molecule fluorescence," ChemPhysChem 6, 2190-2196 (2005).

${ }^{49}$ G. J. Daniels and D. L. Andrews, "Corrected Article: The electronic influence of a third body on resonance energy transfer [J. Chem. Phys. 116, 6701 (2002)]," J. Chem. Phys. 117, 6882-6893 (2002).
${ }^{50}$ G. D. Scholes and D. L. Andrews, "Resonance energy transfer and quantum dots," Phys. Rev. B 72, 125331 (2005).

${ }^{51}$ D. Geissler, N. G. Butlin, D. Hill, H.-G. Löhmannsröben, and N. Hildebrandt, "Multiplexed diagnostics and spectroscopic ruler applications with terbium to quantum dots FRET," 7368, 73680P (2009).

${ }^{52}$ A. Samanta, Y. Zhou, S. Zou, H. Yan, and Y. Liu, "Fluorescence quenching of quantum dots by gold nanoparticles: A potential long range spectroscopic ruler," Nano Lett. 14, 5052-5057 (2014).

${ }^{53}$ F. Morgner, D. Geißler, S. Stufler, N. G. Butlin, H.-G. Löhmannsröben, and N. Hildebrandt, "A quantum-dot-based molecular ruler for multiplexed optical analysis," Angew. Chem., Int. Ed. 49, 7570-7574 (2010).

${ }^{54}$ F. Frezza, L. Pajewski, D. Saccoccioni, and G. Schettini, "Plane-wave expansion of cylindrical functions in lossy media," Opt. Commun. 265, 47-51 (2006).

${ }^{55} \mathrm{R}$. El-Ganainy and S. John, "Resonant dipole-dipole interaction in confined and strong-coupling dielectric geometries," New J. Phys 15, 083033 (2013).

${ }^{56}$ D. L. Dexter, "A theory of sensitized luminescence in solids," J. Chem. Phys. 21, 836-850 (1953).

${ }^{57}$ R. D. Mattuck, A Guide to Feynman Diagrams in the Many-Body Problem (Dover, New York, 1992).

${ }^{58}$ R. P. Feyman, "QED: The strange theory of light and matter" (Princeton University Press, 2006).

${ }^{59}$ Y. Tang and A. E. Cohen, "Optical chirality and its interaction with matter," Phys. Rev. Lett. 104, 163901 (2010).

${ }^{60}$ Y. Tang and A. E. Cohen, "Enhanced enantioselectivity in excitation of chiral molecules by superchiral light," Science 332, 333-336 (2011).

${ }^{61}$ M. M. Coles and D. L. Andrews, "Chirality and angular momentum in optical radiation," Phys. Rev. A 85, 063810 (2012).

${ }^{62}$ M. M. Coles and D. L. Andrews, "Photonic measures of helicity: Optical vortices and circularly polarized reflection," Opt. Lett. 38, 869-871 (2013). 